\title{
Sound Radiation from an Annular Duct with Jet Flow and a Lined Centerbody
}

\author{
A. Demir* \\ Eindhoven University of Technology, 5600 MB Eindhoven, The Netherlands. \\ S.W. Rienstra ${ }^{\dagger}$ \\ Eindhoven University of Technology, 5600 MB Eindhoven, The Netherlands.
}

\begin{abstract}
Analytical solutions for the problem of radiation of sound from a duct with flow have been shown to serve as an important first model for acoustic engine-aircraft engineering applications, useful for understanding the physics and for validating and benchmarking numerical solutions.

The probably most important steps were taken by Levine \& Schwinger and Munt. He showed that application of the Kutta condition, in order to determine the amount of vorticity shed from the trailing edge, goes together with excitation of the Kelvin- Helmholtz instability wave of the jet.

In the present work we will extend this work further by considering the effect of lining on the centerbody and on the afterbody, which is mathematically a much different problem. The lining is of impedance type, while the Ingard-Myers boundary condition is taken to include the effect of the mean flow. The flow is assumed to be subsonic. Differences in the (otherwise uniform) mean flow velocity, density and temperature are taken into account. A vortex sheet which separates the jet from the outer flow emanates from the edge. Due to this velocity discontinuity of the mean flow the jet is unstable. An analytical solution satisfying the full or partial Kutta condition at the trailing edge is found by means of the Wiener-Hopf technique. The jet instability wave is taken apart from the rest of the solution and the effect of applying the Kutta condition to the scattered field is shown, both in far field and near field. The influence of lining on the radiation is displayed graphically.

The problem of a lined afterbody is of particular interest, because its Wiener-Hopf solution embodies a novel application of the so-called "weak factorization".

It should be noted that our primary goal here is to archive the mathematical solution, rather than to unravel all physical possibilities and explore the whole spectrum of problem parameters and their combinations.
\end{abstract}

\section{Introduction}

$\mathrm{T}$ HE problem of radiation of sound from a duct has a long history. Analytical solutions have been shown to be possible only for relatively idealized configurations, for example the seminal Wiener-Hopf solution by Levine and Schwinger for the semi-infinite circular unflanged duct of infinitely thin hard walls. ${ }^{1}$ It was shown by Carrier ${ }^{2}$ that this classical problem without mean flow can be extended to include a subsonic mean flow, although in the case of an exhaust flow the issue of the instability of the jet and the Kutta condition at the trailing edge was only resolved by Munt in. ${ }^{3}$ He showed that application of the Kutta condition, in order to determine the amount of vorticity shed from the trailing edge, goes together with excitation of the Kelvin-Helmholtz instability wave of the jet. This is particularly relevant for numerical solutions in time domain, as these instabilities are practically difficult to avoid. ${ }^{4}$

For many engine-aircraft engineering applications this problem has served as an important first model, useful for understanding the physics and for validating and benchmarking numerical solutions. ${ }^{4-6}$ Exactly for this reason Munt's solution was recently generalized by Gabard e.a. ${ }^{7,8}$ to include a doubly infinite hard-walled centerbody or hub.

In the work of Zhang e.a. ${ }^{6}$ it was found that a relatively important part of the sound radiated from an annular exhaust duct is due to reflection from the afterbody (the outer part of the hub). So it makes sense to apply lining to the hub, in particular the afterbody.

\footnotetext{
${ }^{*}$ Post-doctoral Researcher, Department of Mathematics \& Computer Science, Eindhoven University of Technology, P.O. Box 513, 5600 MB Eindhoven, The Netherlands. AIAA Member.

${ }^{\dagger}$ Associate professor, Department of Mathematics \& Computer Science, Eindhoven University of Technology, P.O. Box 513, 5600 MB Eindhoven, The Netherlands. AIAA Member.
} 
In the present work the radiation of sound out of a semi-infinite annular duct (see Figure 1) with a piecewise uniform jet and co-flow is investigated by means of the Wiener-Hopf technique. The geometry consists of a semiinfinite outer duct with a coaxial infinite lined duct inside. The lining is of impedance type, while the Ingard-Myers ${ }^{9,10}$ boundary condition is taken to include the effect of the mean flow. The flow is assumed to be subsonic. Differences in the (otherwise uniform) mean flow velocity, density and temperature are taken into account. A vortex sheet which separates the jet from the outer flow emanates from the edge. Due to this velocity discontinuity of the mean flow the jet is unstable. An analytical solution satisfying the full or partial Kutta condition at the trailing edge is found by means of the Wiener-Hopf technique. The jet instability wave is taken apart from the rest of the solution and the effect of applying the Kutta condition to the scattered field is shown, both in far field and near field. The influence of the centerbody and lining impedances on the radiation is displayed graphically.

Next we consider the same geometry with only the afterbody lined. In this case the Wiener-Hopf problem is much more complicated, and essentially breaks up into two simultaneous Wiener-Hopf problems. The necessary splitting cannot be performed explicitly, like is the case for the canonical Wiener-Hopf problems. However, by a technique called weak factorization, ${ }^{11,12,14}$ the problem can be reduced to a linear algebraic equation that can be solved efficientlya.

Any instability of the vortex sheet along the lined wall is ignored, and considered as being unphysical. ${ }^{15,16}$

\section{Part I: the lined centerbody}

Consider the geometry which consists of a semi-infinite outer duct and an infinite lined inner duct, i.e. a lined centerbody. Duct walls are assumed to be infinitely thin and they occupy the region $S:\left\{r=R_{1},-\infty<z<\infty\right\} \cup$ $\left\{r=R_{2},-\infty<z<0\right\}$ in circular cylindrical coordinate system $(r, \theta, z)$. Outer duct wall is rigid both from inside and outside while the center body has a lined wall. The liner impedance is characterized by the frequency dependent impedance model $Z=Z(\omega)$. In the region $r>R_{2}$, the ambient flow is uniform and axial with density $\rho_{0}$, velocity $U_{0}$ and speed of sound $c_{0}$. In inner region $R_{1}<r<R_{2}$, there exists a jet which is also uniform and axial with density $\rho_{j}$, velocity $U_{j}$ and speed of sound $c_{j}$. A vortex sheet separates these two different flow at the surface $z>0, r=R_{2}$. Moreover, all quantities are made dimensionless by using outer duct radius and ambient flow properties as a reference

$$
r, z \sim R_{2}, \quad U \sim c_{0}, \quad \rho \sim \rho_{0}, \quad t \sim R_{2} / c_{0}
$$

The velocity potential will be used to obtain the acoustic pressure $p$, velocity $\mathbf{v}$ and density $\rho$ via the following

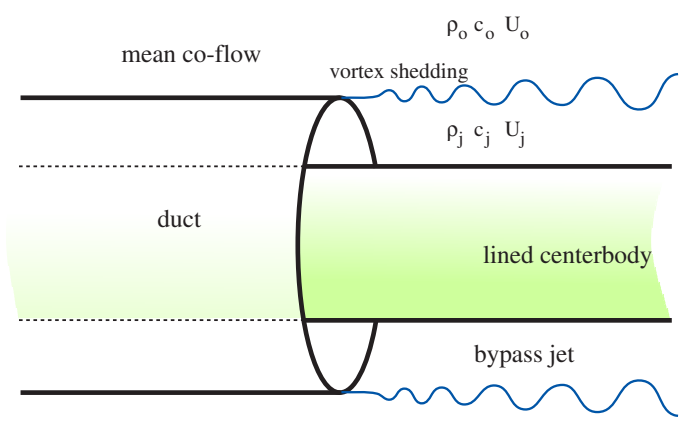

Figure 1. Geometry of annular duct with uniform jet flow and co-flow and lined centerbody

equations

$$
\begin{array}{lllr}
p=-\left(\frac{\partial \phi}{\partial t}+M_{0} \frac{\partial \phi}{\partial z}\right), & \mathbf{v}=\nabla \phi, & \rho=p, & r>1 \\
p=-D_{1}\left(\frac{\partial \phi}{\partial t}+M_{1} \frac{\partial \phi}{\partial z}\right), & \mathbf{v}=\nabla \phi, & \rho=p C_{1}^{2}, & h<r<1
\end{array}
$$

where

$$
M_{0}=U_{0} / c_{0}, \quad M_{1}=U_{j} / c_{0}, \quad C_{1}=c_{0} / c_{j}, \quad D_{1}=\rho_{j} / \rho_{0}, h=R_{1} / R_{2} .
$$

\footnotetext{
${ }^{\mathrm{a}}$ The method is presented in rudimentary form in [13, p.184 section 5.3] but not under this name.
} 
Note that $M_{1}$ is not the local Mach number.

From the symmetry of the geometry and the incident wave, the diffracted field will remain with the same azimuthal and time dependencies as the incident wave. For analysis purposes the total diffracted field can be written in different regions as:

$$
\phi(r, \theta, z, t)=\left\{\begin{array}{lll}
\psi_{1}(r, z) \mathrm{e}^{\mathrm{i} \omega t-\mathrm{i} m \theta} & ; r>1, & z \in(-\infty, \infty) \\
\psi_{2}(r, z) \mathrm{e}^{\mathrm{i} \omega t-\mathrm{i} m \theta}+\psi^{i}(r, z) \mathrm{e}^{\mathrm{i} \omega t-\mathrm{i} m \theta} & ; r \in(h, 1), \quad z \in(-\infty, \infty)
\end{array}\right.
$$

where $\omega$ is the angular frequency and $m$ is the circumferential order.

Time dependency is taken as $\mathrm{e}^{\mathrm{i} \omega t}$ and is suppressed throughout this paper together with the azimuthal dependency $\mathrm{e}^{-\mathrm{i} m \theta}$. Incident field is taken as

$$
\psi^{i}(r, z)=D_{m n}^{+} \Psi_{m n}(r) \mathrm{e}^{-\mathrm{i} \omega \kappa_{m n}^{+} z}
$$

The amplitude of the incident wave, $D_{m n}^{+}$, will be taken equal to 1 in the analysis. $\Psi_{m n}$ is a linear combination of Bessel functions which satisfy boundary conditions on the hard and soft wall:

$$
\Psi_{m n}(r)=\mathcal{Y}_{m}\left(Z, \kappa_{m n}^{+}\right) J_{m}\left(\beta_{m n}^{+} r\right)-\mathscr{g}_{m}\left(Z, \kappa_{m n}^{+}\right) Y_{m}\left(\beta_{m n}^{+} r\right)
$$

where

$$
\begin{aligned}
& \mathscr{g}_{m}(Z, u)=-\frac{\mathrm{i} D_{1}}{Z}\left(1-M_{1} u\right)^{2} J_{m}\left(\lambda_{1} \omega h\right)+\lambda_{1} J_{m}^{\prime}\left(\lambda_{1} \omega h\right), \\
& y_{m}(Z, u)=-\frac{\mathrm{i} D_{1}}{Z}\left(1-M_{1} u\right)^{2} Y_{m}\left(\lambda_{1} \omega h\right)+\lambda_{1} Y_{m}^{\prime}\left(\lambda_{1} \omega h\right) .
\end{aligned}
$$

$Z$ denotes the acoustic impedance of the soft wall and the $\beta_{m n}^{ \pm}$'s are the roots of the equation

$$
\mathcal{y}_{m}\left(Y, \kappa_{m n}^{ \pm}\right) J_{m}^{\prime}\left(\beta_{m n}^{ \pm}\right)-\mathcal{g}_{m}\left(Y, \kappa_{m n}^{ \pm}\right) Y_{m}^{\prime}\left(\beta_{m n}^{ \pm}\right)=0
$$

Derivatives with respect to the argument are denoted by a prime ('), and $\omega \kappa_{m n}^{ \pm}$'s are the axial wavenumbers. The signs $(+)$ and (-) show right and left running modes, respectively. For square root $\kappa_{m n}^{ \pm}$we identify the proper branch by defining

$$
\begin{gathered}
\kappa_{m n}^{+}=\frac{ \pm \sqrt{C_{1}^{2}-\left(1-M_{1}^{2} C_{1}^{2}\right)\left(\beta_{m n}^{+} / \omega\right)^{2}}-M_{1} C_{1}^{2}}{1-M_{1}^{2} C_{1}^{2}} \text { such that } \operatorname{Im}\left(\kappa_{m n}^{+}\right)<0, \\
\kappa_{m n}^{-}=\frac{ \pm \sqrt{C_{1}^{2}-\left(1-M_{1}^{2} C_{1}^{2}\right)\left(\beta_{m n}^{-} / \omega\right)^{2}}-M_{1} C_{1}^{2}}{1-M_{1}^{2} C_{1}^{2}} \text { such that } \operatorname{Im}\left(\kappa_{m n}^{-}\right)>0 .
\end{gathered}
$$

\section{A. Derivation of the Wiener-Hopf system}

The unknown fields $\psi_{1}(r, z)$ and $\psi_{2}(r, z)$ satisfy the convected wave equations

$$
\begin{array}{r}
{\left[\frac{1}{r} \frac{\partial}{\partial r}\left(r \frac{\partial}{\partial r}\right)+\frac{\partial^{2}}{\partial r^{2}}-\frac{m^{2}}{r^{2}}-\left(\mathrm{i} \omega+M_{0} \frac{\partial}{\partial z}\right)^{2}\right] \psi_{1}(r, z)=0} \\
{\left[\frac{1}{r} \frac{\partial}{\partial r}\left(r \frac{\partial}{\partial r}\right)+\frac{\partial^{2}}{\partial r^{2}}-\frac{m^{2}}{r^{2}}-C_{1}^{2}\left(\mathrm{i} \omega+M_{1} \frac{\partial}{\partial z}\right)^{2}\right] \psi_{2}(r, z)=0}
\end{array}
$$

By taking Fourier transforms of these two equations we obtain the following integral representations of the (still) unknown acoustic fields:

$$
\begin{aligned}
& \psi_{1}(r, z)=\frac{\omega}{2 \pi} \int_{\Gamma} A(u) H_{m}^{(2)}\left(\lambda_{0} \omega r\right) \mathrm{e}^{-\mathrm{i} \omega u z} \mathrm{~d} u \\
& \psi_{2}(r, z)=\frac{\omega}{2 \pi} \int_{\Gamma}\left[B(u) J_{m}\left(\lambda_{1} \omega r\right)+C(u) Y_{m}\left(\lambda_{1} \omega r\right)\right] \mathrm{e}^{-\mathrm{i} \omega u z} \mathrm{~d} u
\end{aligned}
$$


where $\Gamma$ is inverse Fourier transform contour and lies on the real line of the complex $u$-plane (See Fig. 15), $J_{m}$ and $Y_{m}$ are the Bessel and Neumann functions of order $m, H_{m}^{(2)}=J_{m}-\mathrm{i} Y_{m}$ is the Hankel function of the second type. $\lambda_{0}$ and $\lambda_{1}$ are square root functions which are defined as

$$
\begin{array}{ll}
\lambda_{0}(u)=\sqrt{\left(1-u M_{0}\right)^{2}-u^{2}}, & \operatorname{Im}\left(\lambda_{0}\right) \leq 0, \\
\lambda_{1}(u)=\sqrt{C_{1}^{2}\left(1-u M_{1}\right)^{2}-u^{2}}, & \operatorname{Im}\left(\lambda_{1}\right) \leq 0 .
\end{array}
$$

Branch cuts for $\lambda_{0}$ are taken on the line from $1 /\left(1+M_{0}\right)$ to $+\infty$ and from $-\infty$ to $-1 /\left(1-M_{0}\right)$. Similarly for $\lambda_{1}$ branch cuts start from $C_{1} /\left(1+M_{1} C_{1}\right)$ to $+\infty$ and from $-\infty$ to $-C_{1} /\left(1-M_{1} C_{1}\right)$.

As usual in this kind of Wiener-Hopf problems, the analysis will be facilitated by giving frequency $\omega$ a small negative imaginary part, written as $\omega=|\omega| \mathrm{e}^{-\mathrm{i} \delta}$. This will lead to an infinite strip $S$ in the complex $u$-plane through the origin, inclined under an angle $\delta$, and just small enough to fit between the branch cuts of $\lambda_{0}$ and $\lambda_{1}$, along which the Wiener-Hopf equation will be formulated. Eventually $\delta \rightarrow 0$ and strip $S$ will merge into the real axis.

Equations for the unknown spectral coefficients $A(u), B(u)$ and $C(u)$ will be obtained below from boundary conditions and relations of continuity.

The Ingard-Myers boundary condition 9,10 of the lined impedance wall with mean flow can be written as

$$
\frac{\partial}{\partial r} \psi_{2}(h, z)=\frac{D_{1}}{\mathrm{i} \omega Z}\left(\mathrm{i} \omega+M_{1} \frac{\partial}{\partial z}\right)^{2} \psi_{2}(h, z), \quad-\infty<z<\infty
$$

The inner and outer sides of the outer duct walls are rigid, so that

$$
\frac{\partial}{\partial r} \psi_{1}(1, z)=\frac{\partial}{\partial r} \psi_{2}(1, z)=0, \quad z<0
$$

By defining the radial displacement of the vortex sheet by $\eta(\theta, z, t)=\xi(z) \mathrm{e}^{\mathrm{i} \omega t-\mathrm{i} m \theta}$, we obtain (cf. [17])

$$
\begin{aligned}
& \left(\mathrm{i} \omega+M_{0} \frac{\partial}{\partial z}\right) \xi(z)=\frac{\partial}{\partial r} \psi_{1}(1, z), \quad z>0, \\
& \left(\mathrm{i} \omega+M_{1} \frac{\partial}{\partial z}\right) \xi(z)=\frac{\partial}{\partial r} \psi_{2}(1, z), \quad z>0 .
\end{aligned}
$$

The pressure is continuous across the vortex sheet, so

$$
D_{1}\left(\mathrm{i} \omega+M_{1} \frac{\partial}{\partial z}\right)\left[\psi_{2}(1, z)+\psi_{i}(1, z)\right]=\left(\mathrm{i} \omega+M_{0} \frac{\partial}{\partial z}\right) \psi_{1}(1, z), \quad z>0
$$

Combining the conditions $(10 \mathrm{~b}, \mathrm{c}, \mathrm{d})$ we have

$$
\begin{aligned}
& \frac{\partial}{\partial r} \psi_{1}(1, z)= \begin{cases}0, & z<0 \\
\left(\mathrm{i} \omega+M_{0} \frac{\partial}{\partial z}\right) \xi(z), & z>0\end{cases} \\
& \frac{\partial}{\partial r} \psi_{2}(1, z)= \begin{cases}0, & z<0 \\
\left(\mathrm{i} \omega+M_{1} \frac{\partial}{\partial z}\right) \xi(z), & z>0\end{cases}
\end{aligned}
$$

In addition to these boundary conditions and continuity relations, we assume that the field radiates outward to infinity and does not reflect backward. A generalized Kutta condition, defining the amount of vorticity shed from the cylinder trailing edge, is also imposed at the edge of the cylinder. The full Kutta condition implies that the pressure is finite at the edge, and the velocity potential is finite and behaves, similar to the vortex sheet displacement, like

$$
\phi(1, z)=\mathcal{O}\left(z^{3 / 2}\right) \quad z \downarrow 0
$$

this condition used for the case of a trailing edge $\left(M_{0}, M_{1}>0\right)$. For leading edge configurations, vortex shedding in the form of a linear perturbation of a uniform mean flow is impossible and we have the usual limited singularity but no-Kutta condition. 
Boundary condition (10a) gives us

$$
C(u)=-\frac{\mathscr{g}_{m}(Z, u)}{\mathcal{y}_{m}(Z, u)} B(u)
$$

By using the conditions (12a,b) and the relation (14a) we can express $A(u)$ and $B(u)$ in terms of an analytic function in the upper half of $u$-plane which are:

$$
\begin{gathered}
A(u) \lambda_{0} H_{m}^{(2) \prime}\left(\lambda_{0} \omega\right)=\mathrm{i}\left(1-u M_{0}\right) F_{+}(u) \\
\frac{B(u)}{y_{m}(Z, u)} \lambda_{1}\left[y_{m}(Z, u) J_{m}^{\prime}\left(\lambda_{1} \omega\right)-\mathcal{g}_{m}(Z, u) Y_{m}^{\prime}\left(\lambda_{1} \omega\right)\right]=\mathrm{i}\left(1-u M_{1}\right) F_{+}(u)
\end{gathered}
$$

where

$$
F_{+}(u)=\int_{0}^{\infty} \xi(z) \mathrm{e}^{\mathrm{i} \omega u z} \mathrm{~d} z
$$

Using continuity of pressure along $r=1, z>0$ we get

$$
\begin{aligned}
\left(1-u M_{0}\right) A(u) H_{m}^{(2)}\left(\lambda_{0} \omega\right)-D_{1}\left(1-u M_{1}\right) \frac{B(u)}{Y_{m}(Z, u)}\left[y_{m}(Z, u) J_{m}\left(\lambda_{1} \omega\right)-\mathscr{g}_{m}(Z, u) Y_{m}\left(\lambda_{1} \omega\right)\right]= \\
G_{-}(u)+\Psi_{m n}(1) D_{1}\left(1-\kappa_{m n}^{+} M_{1}\right) \int_{0}^{\infty} \mathrm{e}^{\mathrm{i} \omega\left(u-\kappa_{m n}^{+}\right) z} \mathrm{~d} z .
\end{aligned}
$$

Upon substitution of $A(u)$ and $B(u)$ into (15), we obtain the following Wiener-Hopf (W-H) equation to be solved

$$
\omega F_{+}(u) K(u)=G_{-}(u)+\Psi_{m n}(1) D_{1} \frac{\left(1-\kappa_{m n}^{+} M_{1}\right)}{\left(\kappa_{m n}^{+}-u\right)} .
$$

$G_{-}(u)$ is a function, analytic in the lower half $u$-plane and defined as

$$
G_{-}(u)=\int_{-\infty}^{0}\left[\left(\mathrm{i} \omega+M_{0} \frac{\partial}{\partial z}\right) \psi_{1}(1, z)-D_{1}\left(\mathrm{i} \omega+M_{1} \frac{\partial}{\partial z}\right) \psi_{2}(1, z)\right] \mathrm{e}^{\mathrm{i} \omega u z} \mathrm{~d} z .
$$

$K(u)$ is the so-called kernel function of the W-H equation.

$$
K(u)=\left\{D_{1}\left(1-u M_{1}\right)^{2} \frac{y_{m}(Z, u) J_{m}\left(\lambda_{1} \omega\right)-g_{m}(Z, u) Y_{m}\left(\lambda_{1} \omega\right)}{\lambda_{1}\left[\mathcal{Y}_{m}(Z, u) J_{m}^{\prime}\left(\lambda_{1} \omega\right)-g_{m}(Z, u) Y_{m}^{\prime}\left(\lambda_{1} \omega\right)\right]}-\left(1-u M_{0}\right)^{2} \frac{H_{m}^{(2)}\left(\lambda_{0} \omega\right)}{\lambda_{0} H_{m}^{(2) \prime}\left(\lambda_{0} \omega\right)}\right\}
$$

\section{B. Kernel function $K(u)$}

Apart from its mathematical importance, the poles and zeros of this function $K(u)$ give us important physical information. The zeros can be thought of coming in two different groups. The poles and a certain amount of zeros are located close to the line $u_{c}=-M_{1} C_{1}^{2} /\left(1-M_{1}^{2} C_{1}^{2}\right)$ which is on the negative side of the real axis. The poles can be found numerically by setting the denominator $\left[\mathcal{Y}_{m}(Z, u) J_{m}^{\prime}\left(\lambda_{1} \omega\right)-\mathscr{g}_{m}(Z, u) Y_{m}^{\prime}\left(\lambda_{1} \omega\right)\right]$ equal to zero. This is also the characteristic equation of the infinite annular duct, so the poles of $K(u)$ correspond to the acoustic modes of the duct. The second group of zeros is more related to the behaviour of the vortex sheet. We can do a high frequency approximation to obtain analytic expressions for this group of zeros of $K(u)$.

In the case ${ }^{18} C_{1}=D_{1}=1$ (cold jet) and $M_{0}=M_{1}=M$ (uniform flow) there is a double zero at $u_{0}=1 / M$. But if $M_{0} \neq M_{1}, u_{0}$ is a simple zero and is found asymptotically as

$$
u_{0}=\left\{\frac{M_{0}+M_{1}}{2}+\frac{1}{2}\left[\left(M_{1}-M_{0}\right)^{2}+4-4 \sqrt{1+\left(M_{1}-M_{0}\right)^{2}}\right]^{1 / 2}\right\}^{-1}
$$

Another important property of this kernel is the behaviour for $|u|$ tending to infinity. As it can be shown shown that

$$
K(u) \sim \mathrm{i} D_{1}\left(1-u M_{1}\right)^{2} / \lambda_{1}-\mathrm{i}\left(1-u M_{0}\right)^{2} / \lambda_{0}
$$

in the strip $S$, it is readily concluded that with mean flow $K(u) \sim \mathcal{O}(u)$, while for $M_{0}=M_{1}=0$, we have $K(u) \sim$ $\mathcal{O}\left(u^{-1}\right)$. 


\section{Factorization of the Kernel}

The crucial step in solving the equation (16a) is to split the kernel function as a ratio of two functions. In the same way as presented in [17], the pole $u=u_{0}$, found in the complex upper half plane, but being associated to the right-running Helmholtz instability of the vortex sheet really belonging to the the lower half plane, is brought to the "other side" by producing regular split functions $\hat{K}_{+}$and $\hat{K}_{-}$and then keeping $K_{+}(u)=\hat{K}_{+}(u)\left(u-u_{0}\right)$ and $K_{-}(u)=\hat{K}_{-}(u)\left(u-u_{0}\right)$ together. In formula this is

$$
K(u)=\frac{K_{+}(u)}{K_{-}(u)}=\frac{\hat{K}_{+}(u)\left(u-u_{0}\right)}{\hat{K}_{-}(u)\left(u-u_{0}\right)}
$$

where $\hat{K}_{+}(u)$ and $\hat{K}_{-}(u)$ are analytic functions in the upper and lower halve $u-$ planes, respectively. By taking a closed contour withing the strip (see $[3,17])$ and using Cauchy's theorem, $\hat{K}_{ \pm}$can be evaluated by the following integrals $^{13}$

$$
\log \hat{K}_{ \pm}(\xi)=\frac{1}{2 \pi \mathrm{i}} \int_{C_{ \pm}} \frac{\log [K(u)]}{u-\xi} \mathrm{d} u .
$$

When $\delta$ is taken to zero, the contours $C_{ \pm}$coincide with the real axis at the respective sides of the branch cuts of $\lambda_{0}$ and $\lambda_{1}$. This is still not a form suitable for numerical evaluation, but more numerical details can be found in the appendix.

\section{Solution of the Wiener-Hopf equation}

Upon substituting this factorization and multiplying (16a) by $K_{-}(u)$ we get

$$
\omega F_{+}(u) K_{+}(u)=K_{-}(u) G_{-}(u)+\Psi_{m n}(1) D_{1} \frac{\left(1-\kappa_{m n}^{+} M_{1}\right) K_{-}(u)}{\left(\kappa_{m n}^{+}-u\right)} .
$$

The second term in the right hand side is analytic on the lower side except for the poles at $u=\kappa_{m n}^{+}$. By subtracting its residue we obtain

$$
\begin{aligned}
\omega F_{+}(u) K_{+}(u)-\Psi_{m n}(1) D_{1}\left(1-\kappa_{m n}^{+} M_{1}\right) & \frac{K_{-}\left(\kappa_{m n}^{+}\right)}{\left(\kappa_{m n}^{+}-u\right)} \\
= & K_{-}(u) G_{-}(u)+\Psi_{m n}(1) D_{1} \frac{\left(1-\kappa_{m n}^{+} M_{1}\right)}{\left(\kappa_{m n}^{+}-u\right)}\left[K_{-}(u)-K_{-}\left(\kappa_{m n}^{+}\right)\right] .
\end{aligned}
$$

Now the left and right hand sides of the equation are analytic in the upper plane and lower plane, respectively, and are identically equal to each other along the real axis (or rather strip $S$, since $\omega$ was temporarily made slightly complex). Therefore, these analytic functions are analytic continuations of each other and they together define an entire function. In the usual way, by using the behaviour of the functions $K_{+}(u), K_{-}(u), G_{-}(u)$ and $F_{+}(u)$ for $|u| \rightarrow \infty$ and Liouville's theorem, it is found that this entire function can only be a constant.

Choosing this constant equal to

$$
E=-\Psi_{m n}(1) D_{1}\left(1-\kappa_{m n}^{+} M_{1}\right) \frac{K_{-}\left(\kappa_{m n}^{+}\right)}{\left(\kappa_{m n}^{+}-u_{0}\right)}(1-\gamma)
$$

we obtain the solution of the W-H equation

$$
F_{+}(u)=-\Psi_{m n}(1) D_{1}\left(1-\kappa_{m n}^{+} M_{1}\right) \frac{K_{-}\left(\kappa_{m n}^{+}\right)}{\omega K_{+}(u)\left(\kappa_{m n}^{+}-u_{0}\right)}\left\{\frac{\left(u-u_{0}\right)}{\left(u-\kappa_{m n}^{+}\right)}-\gamma\right\},
$$

where $\gamma$ is a complex constant related to the Kutta condition, and defined such that

$$
\begin{aligned}
& \gamma=0 \text { for no vortex shedding and no Kutta condition, } \\
& \gamma=1 \text { for just as much vortex shedding as is sufficient for full Kutta condition. }
\end{aligned}
$$

Other values of $\gamma$ will correspond with vortex shedding, but not enough for a full Kutta condition. It seems therefore plausible that any physically reasonable value of $\gamma$ is found for $0 \leq|\gamma| \leq 1$. 


\section{Part II: the lined afterbody}

This part studies the solution for the same problem as before but with a discontinuity at $z=0$ of the impedance boundary condition on the centerbody. Inside the duct, the wall is assumed hard; outside the duct, along what is called the afterbody, the wall is soft. Unlike the first part, this problem results into a Matrix Wiener-Hopf equation which includes a matrix kernel function. A general method for factorizing such a matrix kernel is not known yet. Fortunately, this matrix Wiener-Hopf equation can be reduced into two Wiener-Hopf equations which are solved independently by introducing an infinite sum of poles. The method is called the Weak Factorization method. ${ }^{11-14}$

We write the boundary condition (10a) in two parts (see Figure 2) and introduce a hard-wall mode as incident wave:

$$
\begin{array}{rlrl}
\frac{\partial}{\partial r} \psi_{2}(h, z) & =0, & & z<0, \\
\frac{\partial}{\partial r}\left[\psi_{2}(h, z)+\varphi_{i}(h, z)\right] & =\frac{D_{1}}{\mathrm{i} \omega Z}\left(\mathrm{i} \omega+M_{1} \frac{\partial}{\partial z}\right)^{2}\left[\psi_{2}(h, z)+\varphi_{i}(h, z)\right], & & z>0, \\
\varphi_{i}(r, z) & =E_{m n}^{+} \Xi_{m n}(r) \mathrm{e}^{-\mathrm{i} \omega \mu_{m n}^{+} z}, & & \\
\Xi_{m n}(r) & =Y_{m}^{\prime}\left(\alpha_{m n} h\right) J_{m}\left(\alpha_{m n} r\right)-J_{m}^{\prime}\left(\alpha_{m n} h\right) Y_{m}\left(\alpha_{m n} r\right) . &
\end{array}
$$

Amplitude $E_{m n}^{+}$will be taken equal to 1 in the analysis. Radial wave number $\alpha_{m n}$ is the $n$-th non-trivial zero of $Y_{m}^{\prime}(\alpha h) J_{m}^{\prime}(\alpha)-J_{m}^{\prime}(\alpha h) Y_{m}^{\prime}(\alpha)$. The axial wave number $\mu_{m n}^{ \pm}$is now, for hard walls, expressed as:

$$
\mu_{m n}^{ \pm}= \begin{cases}\frac{ \pm \sqrt{C_{1}^{2}-\left(1-M_{1}^{2} C_{1}^{2}\right) \alpha_{m n}^{2} / \omega^{2}}-M_{1} C_{1}^{2}}{1-M_{1}^{2} C_{1}^{2}}, & C_{1}^{2} \geq\left(1-M_{1}^{2} C_{1}^{2}\right) \alpha_{m n}^{2} / \omega^{2} \quad \text { (cut-on) } \\ \frac{\mp \mathrm{i} \sqrt{\left(1-M_{1}^{2} C_{1}^{2}\right) \alpha_{m n}^{2} / \omega^{2}-C_{1}^{2}}-M_{1} C_{1}^{2}}{1-M_{1}^{2} C_{1}^{2}}, & \text { otherwise (cut off) }\end{cases}
$$

By application of Fourier transformation in $z$ we get obtain

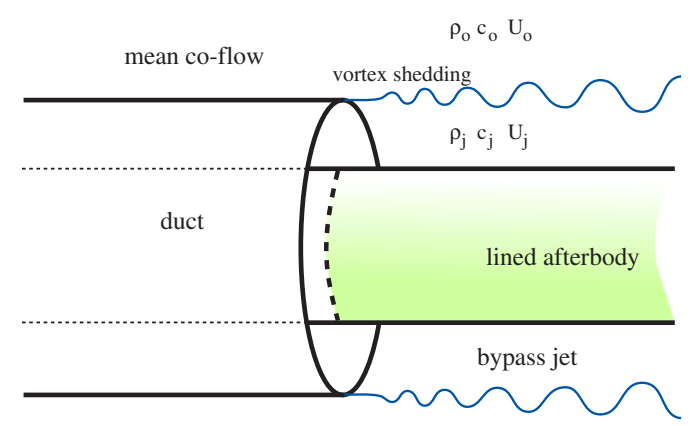

Figure 2. Geometry of annular duct with uniform jet flow and co-flow and lined afterbody

$$
\begin{aligned}
& B(u)=\frac{\pi}{2 \mathrm{i} D_{1}} \frac{h Z}{\left(1-M_{1} u\right)^{2}}\left[\Phi_{1}^{+}(u) y_{m}(Z, u)-\left(\Phi_{1}^{-}(u)-D_{1} \Xi_{m n}(h) \frac{\left(1-\mu_{m n}^{+} M_{1}\right)^{2}}{Z\left(u-\mu_{m n}^{+}\right)}\right) \lambda_{1} Y_{m}^{\prime}\left(\lambda_{1} \omega h\right)\right] \\
& C(u)=-\frac{\pi}{2 \mathrm{i} D_{1}} \frac{h Z}{\left(1-M_{1} u\right)^{2}}\left[\Phi_{1}^{+}(u) g_{m}(Z, u)-\left(\Phi_{1}^{-}(u)-D_{1} \Xi_{m n}(h) \frac{\left(1-\mu_{m n}^{+} M_{1}\right)^{2}}{Z\left(u-\mu_{m n}^{+}\right)}\right) \lambda_{1} J_{m}^{\prime}\left(\lambda_{1} \omega h\right)\right]
\end{aligned}
$$

where $\Xi_{m n}(h)=2 / \pi \alpha_{m n} h$ from the Wronskian for Besselfunctions. $\Phi_{1}^{+}(u)$ and $\Phi_{1}^{-}(u)$ are functions analytic in upper and lower half planes, respectively. Their definitions are:

$$
\begin{aligned}
& \Phi_{1}^{+}(u)=\int_{0}^{\infty} \frac{\partial}{\partial r} \psi_{2}(h, z) \mathrm{e}^{\mathrm{i} \omega u z} \mathrm{~d} z \\
& \Phi_{1}^{-}(u)=\int_{-\infty}^{0}\left[\frac{\partial}{\partial r} \psi_{2}(h, z)-\frac{D_{1}}{\mathrm{i} \omega Z}\left(\mathrm{i} \omega+M_{1} \frac{\partial}{\partial z}\right)^{2} \psi_{2}(h, z)\right] \mathrm{e}^{\mathrm{i} \omega u z} \mathrm{~d} z .
\end{aligned}
$$


Using conditions (11) and (12b) together with (25a,b) we obtain

$$
\begin{aligned}
& \frac{\pi}{2 \mathrm{i} D_{1}} \frac{\lambda_{1} h Z \Phi_{1}^{+}(u)}{\left(1-M_{1} u\right)^{2}}\left[y_{m}(Z, u) J_{m}^{\prime}\left(\lambda_{1} \omega\right)-g_{m}(Z, u) Y_{m}^{\prime}\left(\lambda_{1} \omega\right)\right]-\frac{\pi}{2 \mathrm{i} D_{1}} \frac{\lambda_{1}^{2} h Z}{\left(1-M_{1} u\right)^{2}} \ldots \\
& \quad \ldots\left\{\Phi_{1}^{-}(u)-\frac{2 D_{1}\left(1-\mu_{m n}^{+} M_{1}\right)^{2}}{\pi \alpha_{m n} h Z\left(u-\mu_{m n}^{+}\right)}\right\}\left[Y_{m}^{\prime}\left(\lambda_{1} \omega h\right) J_{m}^{\prime}\left(\lambda_{1} \omega\right)-J_{m}^{\prime}\left(\lambda_{1} \omega h\right) Y_{m}^{\prime}\left(\lambda_{1} \omega\right)\right]=\mathrm{i}\left(1-u M_{1}\right) F_{+}(u)
\end{aligned}
$$

and

$$
\begin{array}{r}
-\omega\left(1-u M_{0}\right)^{2} \frac{H_{m}^{(2)}\left(\lambda_{0} \omega\right)}{\lambda_{0} H_{m}^{(2) \prime}\left(\lambda_{0} \omega\right)} F_{+}(u)-\frac{\pi}{2} \frac{\omega h Z}{\left(1-M_{1} u\right)} \Phi_{1}^{+}(u)\left[y_{m}(Z, u) J_{m}\left(\lambda_{1} \omega\right)-g_{m}(Z, u) Y_{m}\left(\lambda_{1} \omega\right)\right] \\
+\frac{\pi}{2} \frac{\omega h Z}{\left(1-M_{1} u\right)}\left\{\Phi_{1}^{-}(u)-\frac{2 D_{1}\left(1-\mu_{m n}^{+} M_{1}\right)^{2}}{\pi \alpha_{m n} h Z\left(u-\mu_{m n}^{+}\right)}\right\} \lambda_{1}\left[Y_{m}^{\prime}\left(\lambda_{1} \omega h\right) J_{m}\left(\lambda_{1} \omega\right)-J_{m}^{\prime}\left(\lambda_{1} \omega h\right) Y_{m}\left(\lambda_{1} \omega\right)\right] \\
=G_{-}(u)+\mathrm{i} \omega \Xi_{m n}(1) D_{1}\left(1-\mu_{m n}^{+} M_{1}\right) \int_{0}^{\infty} \mathrm{e}^{\mathrm{i} \omega\left(u-\mu_{m n}^{+}\right) z} \mathrm{~d} z
\end{array}
$$

Eliminating $\Phi_{1}^{+}(u)$ in the latter equation with the help of the first equation, we reduce (26a,b) into two simultaneous Wiener-Hopf equations:

$$
\begin{array}{r}
\Phi_{1}^{+}(u)-\lambda_{1} \frac{Y_{m}^{\prime}\left(\lambda_{1} \omega h\right) J_{m}^{\prime}\left(\lambda_{1} \omega\right)-J_{m}^{\prime}\left(\lambda_{1} \omega h\right) Y_{m}^{\prime}\left(\lambda_{1} \omega\right)}{y_{m}(Z, u) J_{m}^{\prime}\left(\lambda_{1} \omega\right)-\mathcal{g}_{m}(Z, u) Y_{m}^{\prime}\left(\lambda_{1} \omega\right)}\left\{\Phi_{1}^{-}(u)-\frac{2 D_{1}\left(1-\mu_{m n}^{+} M_{1}\right)^{2}}{\pi \alpha_{m n} h Z\left(u-\mu_{m n}^{+}\right)}\right\} \\
=-\frac{2}{\pi} \frac{D_{1}\left(1-M_{1} u\right)^{3} F_{+}(u)}{h Z \lambda_{1}\left[y_{m}(Z, u) J_{m}^{\prime}\left(\lambda_{1} \omega\right)-g_{m}(Z, u) Y_{m}^{\prime}\left(\lambda_{1} \omega\right)\right]}
\end{array}
$$

and

$$
\begin{aligned}
& \omega F_{+}(u)\left[D_{1}\left(1-u M_{1}\right)^{2} \frac{y_{m}(Z, u) J_{m}\left(\lambda_{1} \omega\right)-g_{m}(Z, u) Y_{m}\left(\lambda_{1} \omega\right)}{\lambda_{1}\left[y_{m}(Z, u) J_{m}^{\prime}\left(\lambda_{1} \omega\right)-g_{m}(Z, u) Y_{m}^{\prime}\left(\lambda_{1} \omega\right)\right]}-\left(1-u M_{0}\right)^{2} \frac{H_{m}^{(2)}\left(\lambda_{0} \omega\right)}{\lambda_{0} H_{m}^{(2) \prime}\left(\lambda_{0} \omega\right)}\right] \\
& +\frac{2 \mathrm{i}}{\pi \omega} \frac{D_{1}\left(1-M_{1} u\right)}{\lambda_{1}\left[\mathcal{y}_{m}(Z, u) J_{m}^{\prime}\left(\lambda_{1} \omega\right)-\mathcal{g}_{m}(Z, u) Y_{m}^{\prime}\left(\lambda_{1} \omega\right)\right]}\left\{\Phi_{1}^{-}(u)-\frac{2 D_{1}\left(1-\mu_{m n}^{+} M_{1}\right)^{2}}{\pi \alpha_{m n} h Z\left(u-\mu_{m n}^{+}\right)}\right\} \\
& =G_{-}(u)+\mathrm{i} \omega \Xi_{m n}(1) D_{1}\left(1-\mu_{m n}^{+} M_{1}\right) \int_{0}^{\infty} \mathrm{e}^{\mathrm{i} \omega\left(u-\mu_{m n}^{+}\right) z} \mathrm{~d} z
\end{aligned}
$$

This set of equations will be solved in the next section.

\section{A. Wiener-Hopf Solution: Weak Factorization}

First consider the equation

$$
\begin{gathered}
\Phi_{1}^{+}(u)-N(u)\left\{\Phi_{1}^{-}(u)-\frac{2 D_{1}\left(1-\mu_{m n}^{+} M_{1}\right)^{2}}{\pi \alpha_{m n} h Z\left(u-\mu_{m n}^{+}\right)}\right\}=-\frac{2}{\pi} \frac{D_{1}\left(1-M_{1} u\right)^{3} F_{+}(u)}{h Z \lambda_{1}\left[\mathcal{y}_{m}(Z, u) J_{m}^{\prime}\left(\lambda_{1} \omega\right)-\mathcal{g}_{m}(Z, u) Y_{m}^{\prime}\left(\lambda_{1} \omega\right)\right]} \\
N(u)=\frac{\lambda_{1}\left[Y_{m}^{\prime}\left(\lambda_{1} \omega h\right) J_{m}^{\prime}\left(\lambda_{1} \omega\right)-J_{m}^{\prime}\left(\lambda_{1} \omega h\right) Y_{m}^{\prime}\left(\lambda_{1} \omega\right)\right]}{\left[\mathcal{y}_{m}(Z, u) J_{m}^{\prime}\left(\lambda_{1} \omega\right)-g_{m}(Z, u) Y_{m}^{\prime}\left(\lambda_{1} \omega\right)\right]}
\end{gathered}
$$

where $N(u)$ is a meromorphic function and can be written as the ratio of two functions which are analytic in the upper and lower half of $u$-plane:

$$
N(u)=\frac{N_{+}(u)}{N_{-}(u)} .
$$

The $N_{ \pm}(u)$ functions can be calculated numerically by using the same procedure in calculating $K_{ \pm}(u)$, while their behaviour for $|u| \rightarrow \infty$ is given by

$$
N_{+}(u) \sim u^{-1 / 2}, \quad N_{-}(u) \sim u^{1 / 2}
$$


Multiplying this equation by $1 / N_{+}(u)$ yields

$$
\begin{aligned}
& \frac{\Phi_{1}^{+}(u)}{N_{+}(u)}+\frac{2\left(1-M_{1} u\right)^{3}}{\pi h Z} \frac{D_{1} F_{+}(u)}{\lambda_{1}\left[\mathcal{Y}_{m}(Z, u) J_{m}^{\prime}\left(\lambda_{1} \omega\right)-g_{m}(Z, u) Y_{m}^{\prime}\left(\lambda_{1} \omega\right)\right] N_{+}(u)} \\
& +\frac{2 D_{1}\left(1-\mu_{m n}^{+} M_{1}\right)^{2}}{\pi \alpha_{m n} h Z N_{-}\left(\mu_{m n}^{+}\right)\left(u-\mu_{m n}^{+}\right)}=\frac{1}{N_{-}(u)}\left\{\Phi_{1}^{-}(u)-\frac{2 D_{1}\left(1-\mu_{m n}^{+} M_{1}\right)^{2}}{\pi \alpha_{m n} h Z\left(u-\mu_{m n}^{+}\right)}\right\}+\frac{2 D_{1}\left(1-\mu_{m n}^{+} M_{1}\right)^{2}}{\pi \alpha_{m n} h Z N_{-}\left(\mu_{m n}^{+}\right)\left(u-\mu_{m n}^{+}\right)} .
\end{aligned}
$$

The right-hand side is analytic on the lower half-plane but the analyticity of the left hand side is violated by the zeros of denominator at the upper half-plane, namely

$$
\lambda_{1}\left\{y_{m}\left(Z, \kappa_{m n}^{-}\right) J_{m}^{\prime}\left[\lambda_{1}\left(\kappa_{m n}^{-}\right) \omega\right]-\mathscr{g}_{m}\left(Z, \kappa_{m n}^{-}\right) Y_{m}^{\prime}\left[\lambda_{1}\left(\kappa_{m n}^{-}\right) \omega\right]\right\}=0 .
$$

We have to subtract the residue contributions from these poles (which is called Weak Factorization), to get

$$
\begin{gathered}
\frac{\Phi_{1}^{+}(u)}{N_{+}(u)}+\frac{2\left(1-M_{1} u\right)^{3}}{\pi h Z} \frac{D_{1} F_{+}(u)}{\lambda_{1}\left[y_{m}(Z, u) J_{m}^{\prime}\left(\lambda_{1} \omega\right)-g_{m}(Z, u) Y_{m}^{\prime}\left(\lambda_{1} \omega\right)\right] N_{+}(u)} \\
\quad+\frac{2 D_{1}\left(1-\mu_{m n}^{+} M_{1}\right)^{2}}{\pi \alpha_{m n} h Z N_{-}\left(\mu_{m n}^{+}\right)\left(u-\mu_{m n}^{+}\right)}-\sum_{p=1}^{\infty} \frac{a_{m p}}{u-\kappa_{m p}^{-}} \\
=\frac{1}{N_{-}(u)}\left\{\Phi_{1}^{-}(u)-\frac{2 D_{1}\left(1-\mu_{m n}^{+} M_{1}\right)^{2}}{\pi \alpha_{m n} h Z\left(u-\mu_{m n}^{+}\right)}\right\}+\frac{2 D_{1}\left(1-\mu_{m n}^{+} M_{1}\right)^{2}}{\pi \alpha_{m n} h Z N_{-}\left(\mu_{m n}^{+}\right)\left(u-\mu_{m n}^{+}\right)}-\sum_{p=1}^{\infty} \frac{a_{m p}}{u-\kappa_{m p}^{-}}
\end{gathered}
$$

where

$$
a_{m p}=\frac{2\left(1-M_{1} \kappa_{m p}^{-}\right)^{3}}{\pi h Z} \frac{D_{1} F_{+}\left(\kappa_{m p}^{-}\right)}{N_{+}\left(\kappa_{m p}^{-}\right) \lambda_{1}\left(\kappa_{m p}^{-}\right)\left[y_{m}(Z, u) J_{m}^{\prime}\left(\lambda_{1} \omega\right)-g_{m}(Z, u) Y_{m}^{\prime}\left(\lambda_{1} \omega\right)\right]_{u=\kappa_{m p}^{\prime}}^{\prime}}
$$

Then, utilizing Liouville's theorem, the solution is found as follows

$$
\begin{aligned}
& \frac{1}{N_{-}(u)}\left\{\Phi_{1}^{-}(u)-\frac{2 D_{1}\left(1-\mu_{m n}^{+} M_{1}\right)^{2}}{\pi \alpha_{m n} h Z\left(u-\mu_{m n}^{+}\right)}\right\}=\sum_{p=1}^{\infty} \frac{a_{m p}}{u-\kappa_{m p}^{-}}-\frac{2 D_{1}\left(1-\mu_{m n}^{+} M_{1}\right)^{2}}{\pi \alpha_{m n} h Z\left(u-\mu_{m n}^{+}\right) N_{-}\left(\mu_{m n}^{+}\right)} \\
& \frac{\Phi_{1}^{+}(u)}{N_{+}(u)}+\frac{2\left(1-M_{1} u\right)^{3}}{\pi h Z} \frac{D_{1} F_{+}(u)}{\lambda_{1}\left[y_{m}(Z, u) J_{m}^{\prime}\left(\lambda_{1} \omega\right)-g_{m}(Z, u) Y_{m}^{\prime}\left(\lambda_{1} \omega\right)\right] N_{+}(u)} \\
& +\frac{2 D_{1}\left(1-\mu_{m n}^{+} M_{1}\right)^{2}}{\pi \alpha_{m n} h Z N_{-}\left(\mu_{m n}^{+}\right)\left(u-\mu_{m n}^{+}\right)}=\sum_{p=1}^{\infty} \frac{a_{m p}}{u-\kappa_{m p}^{-}}
\end{aligned}
$$

The second Wiener-Hopf equation has the same kernel as the lined centerbody problem. We therefore follow the same procedure as before

$$
\begin{aligned}
\omega F_{+}(u) K(u)+\frac{2 i}{\pi \omega} \frac{D_{1}\left(1-u M_{1}\right)}{\lambda_{1}\left[y_{m}(Z, u) J_{m}^{\prime}\left(\lambda_{1} \omega\right)-g_{m}(Z, u) Y_{m}^{\prime}\left(\lambda_{1} \omega\right)\right]}\left\{\begin{aligned}
\left.\Phi_{1}^{-}(u)-\frac{2 D_{1}\left(1-\mu_{m n}^{+} M_{1}\right)^{2}}{\pi \alpha_{m n} h Z\left(u-\mu_{m n}^{+}\right)}\right\} \\
=G_{-}(u)-\Xi_{m n}(1) D_{1} \frac{\left(1-\mu_{m n}^{+} M_{1}\right)}{\left(u-\mu_{m n}^{+}\right)}
\end{aligned}\right.
\end{aligned}
$$

First multiply the equation by $K_{-}(u)$

$$
\begin{array}{r}
\omega F_{+}(u) K_{+}(u)+\frac{2 i}{\pi \omega} \frac{K_{-}(u) D_{1}\left(1-u M_{1}\right)}{\lambda_{1}\left[\mathcal{Y}_{m}(Z, u) J_{m}^{\prime}\left(\lambda_{1} \omega\right)-g_{m}(Z, u) Y_{m}^{\prime}\left(\lambda_{1} \omega\right)\right]}\left\{\Phi_{1}^{-}(u)-\frac{2 D_{1}\left(1-\mu_{m n}^{+} M_{1}\right)^{2}}{\pi \alpha_{m n} h Z\left(u-\mu_{m n}^{+}\right)}\right\} \\
=K_{-}(u) G_{-}(u)-\Xi_{m n}(1) D_{1} \frac{\left(1-\mu_{m n}^{+} M_{1}\right)}{\left(u-\mu_{m n}^{+}\right)} K_{-}(u)
\end{array}
$$


and we again see that the regularity of left hand side is violated by the zeros on the lower half plane.

$$
\lambda_{1}\left\{y_{m}\left(Z, \kappa_{m n}^{+}\right) J_{m}^{\prime}\left[\lambda_{1}\left(\kappa_{m n}^{+}\right) \omega\right]-\mathscr{g}_{m}\left(Z, \kappa_{m n}^{+}\right) Y_{m}^{\prime}\left[\lambda_{1}\left(\kappa_{m n}^{+}\right) \omega\right]\right\}=0 .
$$

Subtracting their residue contributions yields

$$
\begin{aligned}
& \omega F_{+}(u) K_{+}(u)-\sum_{p=1}^{\infty} \frac{b_{m p}}{u-\kappa_{m p}^{+}}=K_{-}(u) G_{-}(u)-\Xi_{m n}(1) D_{1} \frac{\left(1-\mu_{m n}^{+} M_{1}\right)}{\left(u-\mu_{m n}^{+}\right)} K_{-}(u) \\
& \quad-\frac{2 \mathrm{i}}{\pi \omega} \frac{K_{-}(u) D_{1}\left(1-u M_{1}\right)}{\lambda_{1}\left[y_{m}(Z, u) J_{m}^{\prime}\left(\lambda_{1} \omega\right)-g_{m}(Z, u) Y_{m}^{\prime}\left(\lambda_{1} \omega\right)\right]}\left\{\Phi_{1}^{-}(u)-\frac{2 D_{1}\left(1-\mu_{m n}^{+} M_{1}\right)^{2}}{\pi \alpha_{m n} h Z\left(u-\mu_{m n}^{+}\right)}\right\}-\sum_{p=1}^{\infty} \frac{b_{m p}}{u-\kappa_{m p}^{+}}
\end{aligned}
$$

where

$$
b_{m p}=-\frac{2 i}{\pi \omega} \frac{D_{1}\left(1-\kappa_{m p}^{+} M_{1}\right) K_{-}\left(\kappa_{m p}^{+}\right)}{\lambda_{1}\left(\kappa_{m p}^{+}\right)\left[y_{m}(Z, u) J_{m}^{\prime}\left(\lambda_{1} \omega\right)-g_{m}(Z, u) Y_{m}^{\prime}\left(\lambda_{1} \omega\right)\right]_{u=\kappa_{m p}^{\prime}}^{\prime}}\left\{\Phi_{1}^{-}\left(\kappa_{m p}^{+}\right)-\frac{2 D_{1}\left(1-\mu_{m n}^{+} M_{1}\right)^{2}}{\pi \alpha_{m n} h Z\left(\kappa_{m p}^{+}-\mu_{m n}^{+}\right)}\right\} .
$$

We end up with the formal solution

$$
\begin{gathered}
\omega F_{+}(u) K_{+}(u)=\sum_{p=1}^{\infty} \frac{b_{m p}}{\kappa_{m p}^{+}-u_{0}}\left[\frac{u-u_{0}}{u-\kappa_{m p}^{+}}-\gamma\right] \\
K_{-}(u) G_{-}(u)=\Xi_{m n}(1) D_{1} \frac{\left(1-\mu_{m n}^{+} M_{1}\right)}{\left(u-\mu_{m n}^{+}\right)} K_{-}(u) \\
+\frac{2 \mathrm{i}}{\pi \omega} \frac{K_{-}(u) D_{1}\left(1-u M_{1}\right)}{\lambda_{1}\left[\mathcal{Y}_{m}(Z, u) J_{m}^{\prime}\left(\lambda_{1} \omega\right)-\mathscr{g}_{m}(Z, u) Y_{m}^{\prime}\left(\lambda_{1} \omega\right)\right]}\left\{\Phi_{1}^{-}(u)-\frac{2 D_{1}\left(1-\mu_{m n}^{+} M_{1}\right)^{2}}{\pi \alpha_{m n} h Z\left(u-\mu_{m n}^{+}\right)}\right\}+\sum_{p=1}^{\infty} \frac{b_{m p}}{u-\kappa_{m p}^{+}}
\end{gathered}
$$

These solutions involve undetermined coefficients $a_{n}$ and $b_{n}$, which can be determined by solving the following linear algebraic system numerically.

$$
\begin{gathered}
\frac{1}{2} \pi \mathrm{i} \omega b_{m r} \frac{\lambda_{1}\left(\kappa_{m r}^{+}\right)}{D_{1}\left(1-\kappa_{m r}^{+} M_{1}\right) K_{-}\left(\kappa_{m r}^{+}\right) N_{-}\left(\kappa_{m r}^{+}\right)}\left[y_{m}(Z, u) J_{m}^{\prime}\left(\lambda_{1} \omega\right)-g_{m}(Z, u) Y_{m}^{\prime}\left(\lambda_{1} \omega\right)\right]_{u=\kappa_{m r}^{+}}^{\prime} \\
=\sum_{p=1}^{\infty} \frac{a_{m p}}{\kappa_{m r}^{+}-\kappa_{m p}^{-}}-\frac{2 D_{1}\left(1-\mu_{m n}^{+} M_{1}\right)^{2}}{\pi \alpha_{m n} h Z\left(\kappa_{m r}^{+}-\mu_{m n}^{+}\right) N_{-}\left(\mu_{m n}^{+}\right)} \\
\begin{array}{r}
\frac{1}{2} \pi \omega h Z a_{m r} \frac{K_{+}\left(\kappa_{m r}^{-}\right) N_{+}\left(\kappa_{m r}^{-}\right) \lambda_{1}\left(\kappa_{m r}^{-}\right)}{D_{1}\left(1-M_{1} \kappa_{m r}^{-}\right)^{3}}\left[y_{m}(Z, u) J_{m}^{\prime}\left(\lambda_{1} \omega\right)-g_{m}(Z, u) Y_{m}^{\prime}\left(\lambda_{1} \omega\right)\right]_{u=\kappa_{m r}^{-}}^{\prime} \\
=\sum_{p=1}^{\infty} \frac{b_{m p}}{\kappa_{m p}^{+}-u_{0}}\left[\frac{\kappa_{m r}^{-}-u_{0}}{\kappa_{m r}^{-}-\kappa_{m p}^{+}}-\gamma\right]
\end{array}
\end{gathered}
$$

The infinite sums in the equations converge very rapidly so they can be truncated quickly.

\section{Far Field}

The solution in the far field for pressure can be calculated in terms of the following integral

$$
p_{1}(r, z)=\frac{\omega^{2}}{2 \pi} \int_{\Gamma} \frac{\left(1-u M_{0}\right)^{2} F_{+}(u)}{\lambda_{0}} \frac{H_{m}^{(2)}\left(\lambda_{0} \omega r\right)}{H_{m}^{(2) \prime}\left(\lambda_{0} \omega\right)} \mathrm{e}^{-\mathrm{i} \omega u z} \mathrm{~d} u
$$

where $\Gamma$ is the inverse Fourier transform contour. 
For the far field pressure $(r \gg 1)$ we can use the asymptotic formula for the Hankel function

$$
H_{m}^{(2)}\left(\lambda_{0} \omega r\right) \sim \sqrt{\frac{2}{\pi \lambda_{0} \omega r}} \mathrm{e}^{-\mathrm{i}\left(\lambda_{0} \omega r-\frac{1}{2} m \pi-\frac{1}{4} \pi\right)} .
$$

By replacing (41) in the integral given with (40) we get

$$
p_{1}(r, z) \sim \frac{\omega^{2}}{2 \pi} \int_{\Gamma} \frac{\left(1-u M_{0}\right)^{2} F_{+}(u)}{\lambda_{0} H_{m}^{(2) \prime}\left(\lambda_{0} \omega\right)} \sqrt{\frac{2}{\pi \lambda_{0} \omega r}} \mathrm{e}^{-\mathrm{i}\left(\omega \lambda_{0} r+\omega u z-\frac{1}{2} m \pi-\frac{1}{4} \pi\right)} \mathrm{d} u
$$

After transforming the free variables and the integration contour as follows

$$
r=R \sin \theta\left(1-M_{0}^{2}\right)^{1 / 2}, \quad z=R \cos \theta\left(1-M_{0}^{2}\right),
$$

deforming $\Gamma$ into a branch of a hyperbola $\bar{\Gamma}$, we can evaluate the integral by the method of steepest descent

$$
p_{1}(r, z) \sim \frac{\mathrm{i} \omega^{2}}{2 \pi} \int_{\bar{\Gamma}} \frac{\left(1-u M_{0}\right)^{2} F_{+}(u)}{\left(1-M_{0}^{2}\right) \lambda_{0} H_{m}^{(2) \prime}\left(\lambda_{0} \omega\right)} \sqrt{\frac{2 \sin (\theta-\mathrm{i} \tau)}{\pi \omega R \sin \theta}} \mathrm{e}^{-\mathrm{i}\left(\omega R \cosh \tau-\omega R M_{0} \cos \theta-\frac{1}{2} m \pi-\frac{1}{4} \pi\right)} \mathrm{d} \tau .
$$

This means that the major contribution to the integral comes from the vicinity of "saddle point" $\tau_{s}$. This point can be determined via the function $q(\tau)$,

$$
q(\tau)=-\mathrm{i} \cosh \tau, \quad q^{\prime}(\tau)=-\mathrm{i} \sinh \tau, \quad q^{\prime}(0)=0, \quad \tau_{s}=0,
$$

where $q(\tau)$ is an analytic function. Hence the solution in the far field can be approximated by

$$
p_{1}(R, \theta) \sim \frac{\mathrm{i} \omega^{2}}{\pi} \frac{\left(1-u M_{0}\right)^{2} F_{+}(u)}{\left(1-M_{0}^{2}\right)^{1 / 2} \sin \theta H_{m}^{(2) \prime}\left(\lambda_{0} \omega\right)} \mathrm{e}^{\mathrm{i} \frac{1}{2} m \pi} \frac{\mathrm{e}^{-\mathrm{i} \omega R\left(1-M_{0} \cos \theta\right)}}{\omega R} .
$$

After converting the coordinate variables to

$$
z=\bar{R} \cos \bar{\theta}, \quad r=\bar{R} \sin \bar{\theta}
$$

we obtain

$$
p_{1}(\bar{R}, \bar{\theta}) \sim \frac{D_{p}(\bar{\theta})}{\bar{R}} \mathrm{e}^{-\mathrm{i} \omega \bar{R}\left[-M_{0} \cos \bar{\theta}+\left(1-M_{0}^{2} \sin ^{2} \bar{\theta}\right)^{1 / 2}\right] /\left(1-M_{0}^{2}\right)+\mathrm{i} \frac{1}{2}(m+1) \pi}
$$

where directivity $D_{p}$ of the pressure field is given by

$$
D_{p}(\bar{\theta})=\frac{\omega}{\pi} \frac{\left(1-u^{\prime} M_{0}\right)^{2} F_{+}\left(u^{\prime}\right)}{\sin \bar{\theta} H_{m}^{(2) \prime}\left(\lambda_{0}^{\prime} \omega\right)}
$$

$u^{\prime}$ and $\lambda_{0}^{\prime}$ are defined as

$$
u^{\prime}=\frac{\cos \bar{\theta}\left(1-M_{0}^{2} \sin ^{2} \bar{\theta}\right)^{-1 / 2}-M_{0}}{\left(1-M_{0}^{2}\right)}, \quad \lambda_{0}^{\prime}=\frac{\sin \bar{\theta}}{\left(1-M_{0}^{2} \sin ^{2} \bar{\theta}\right)^{1 / 2}}
$$

\section{Numerical Examples}

A series of examples are numerically evaluated to see the effect of the lined versus hard-walled hub, with and without mean flow and Kutta condition. These examples are not meant to be more than an illustration of the found solutions. It is not possible to make an exhaustive investigation of all possible combinations of problem parameters nor scan all the physical consequences. 
The problem parameters that were used are:

$$
\begin{aligned}
\omega & =2 \pi 660 R_{2} / c_{0}=15.0 \text { and } 2 \pi 1095 R_{2} / c_{0}=25.0 \\
m & =4 \\
M_{0} & =U_{0} / c_{0}=99 / 330=0.300 \\
M_{1} & =U_{j} / c_{0}=175 / 330=0.530 \\
M_{j} & =U_{j} / c_{j}=175 / 350=0.500 \\
C_{1} & =c_{0} / c_{j}=330 / 350=0.943 \\
D_{1} & =\rho_{j} / \rho_{0}=1.17 / 1.20=0.975 \\
h & =R_{1} / R_{2}=0.80 / 1.20=0.667 \\
Z & =2-\mathrm{i}
\end{aligned}
$$

For zero flow, the outer and jet properties are the same, where outer flow values are used in both regions. The far field values are plotted dimensionally, at a distance $46.0 \mathrm{~m}$ away from the exhaust plane. In all cases the first, second or third radial mode is taken, with amplitude such that the cross-sectional averaged intensity at $z=0$ is $1 \mathrm{~W} / \mathrm{m}^{2}$.

\section{A. Hard vs soft walls}

In this section we compare hard against lined centerbody. It is seen that the sharply defined lobes, that exist for hard walls, are smoothed out. The effect of the Kutta condition is for both cases about the same. Attenuation is mainly obtained off the main lobe. Remarkably, in singular cases the upstream field with lining can be slightly louder than without.

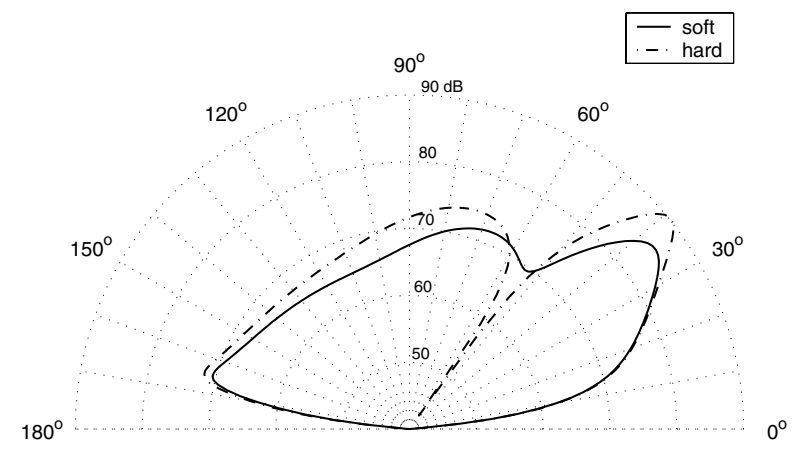

(a) no Kutta condition

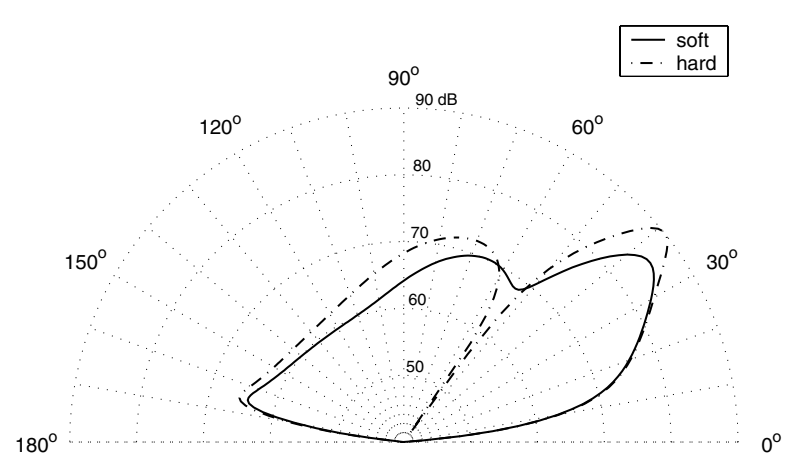

(b) with Kutta condition

Figure 3. Hard vs soft for 1st radial mode with $\omega=15$

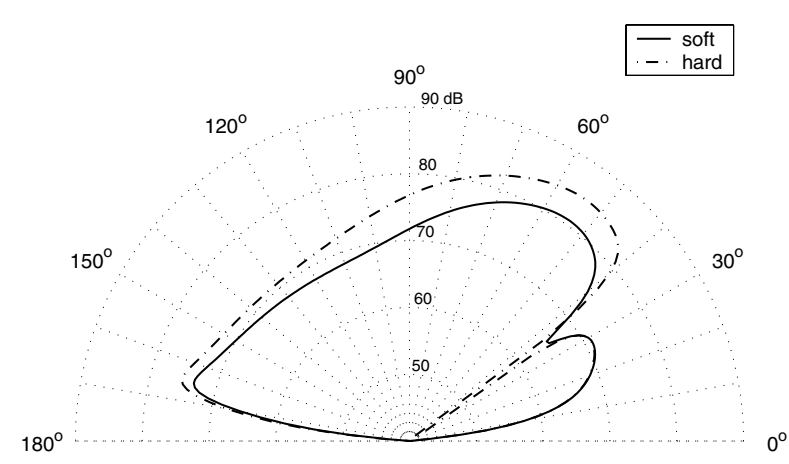

(a) no Kutta condition

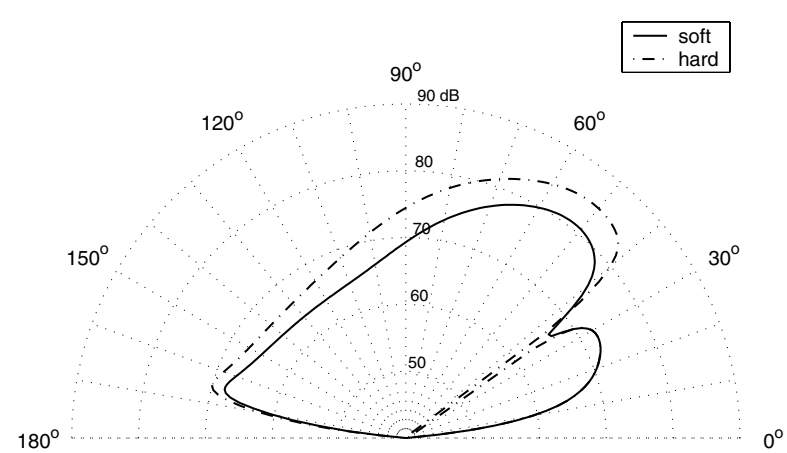

(b) with Kutta condition

Figure 4. Hard vs soft for 2nd radial mode with $\omega=15$ 


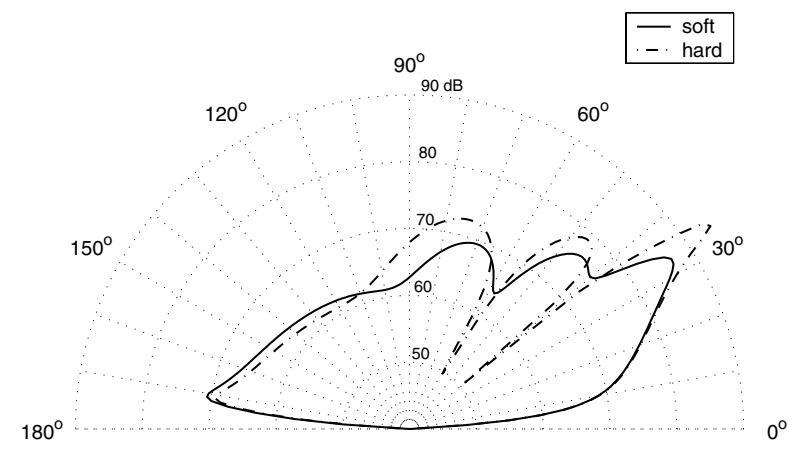

(a) no Kutta condition

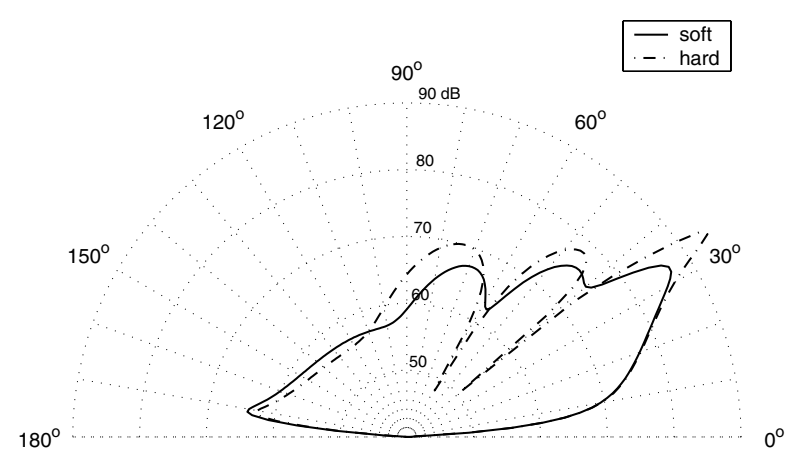

(b) with Kutta condition

Figure 5. Hard vs soft for 1st radial mode with $\omega=25$

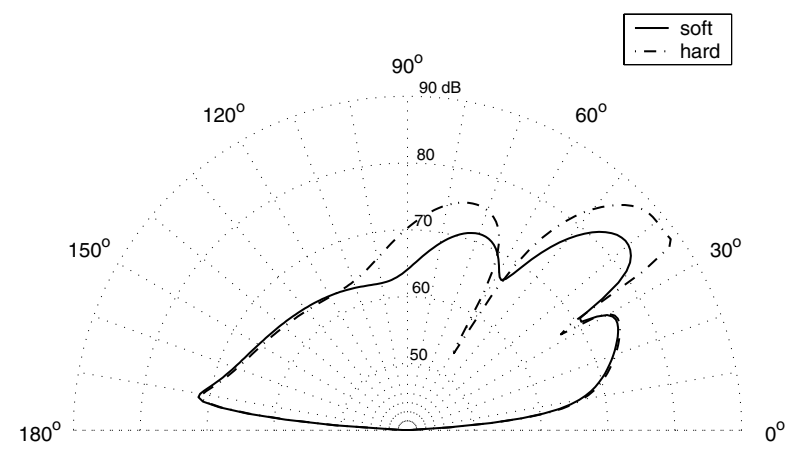

(a) no Kutta condition

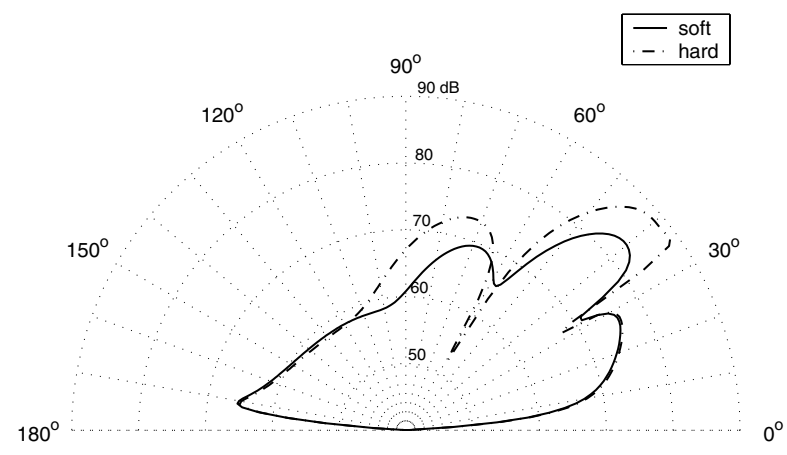

(b) with Kutta condition

Figure 6. Hard vs soft for 2 nd radial mode with $\omega=25$

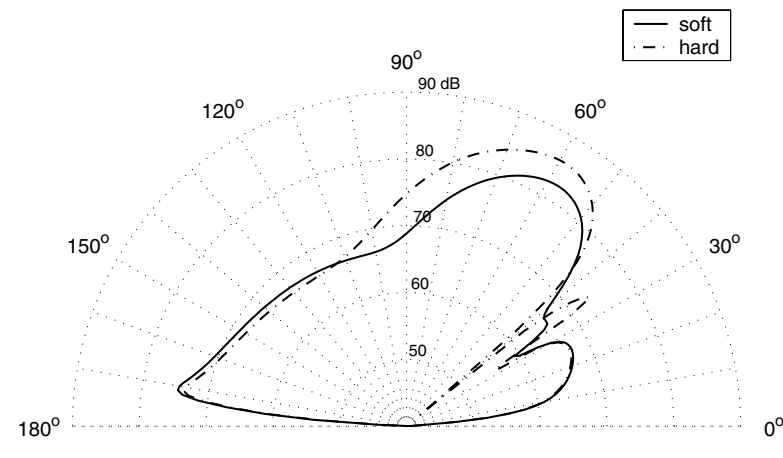

(a) no Kutta condition

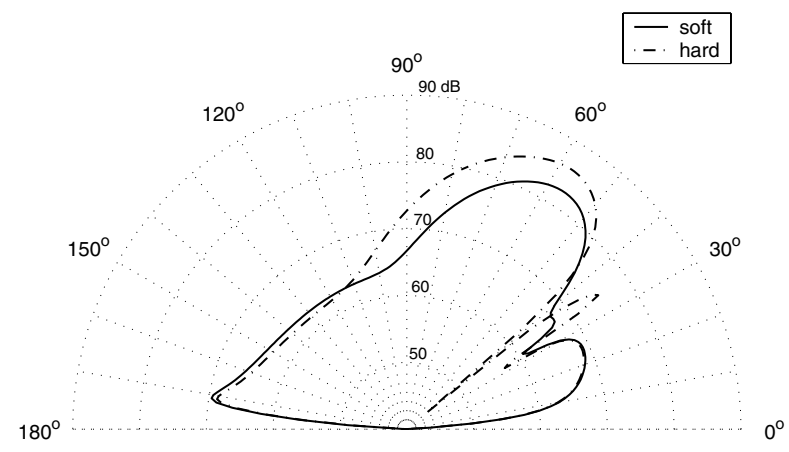

(b) with Kutta condition

Figure 7. Hard vs soft for 3d radial mode with $\omega=25$

\section{B. With vs without mean flow}

In this section we compare the effect of mean flow against no mean flow, in the presence of a lined centerbody. In the downstream arc, the effect of the shear layer refraction is always visible. The effect of the Kutta condition is with these relatively high frequencies only small, and limited to upstream angels. 


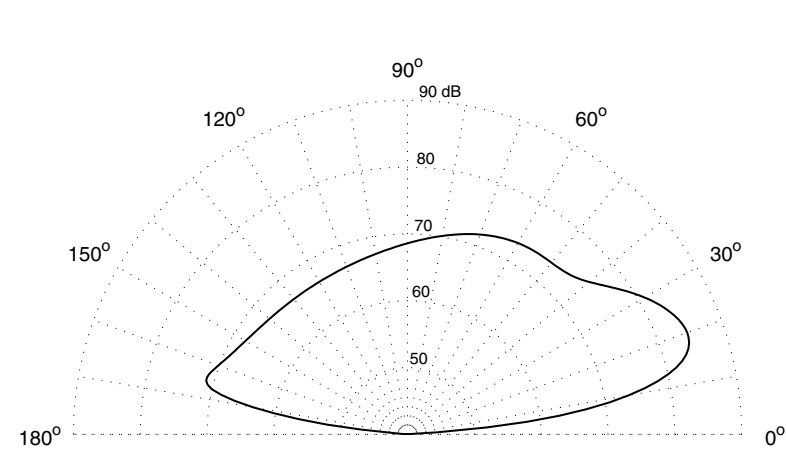

(a) no mean flow

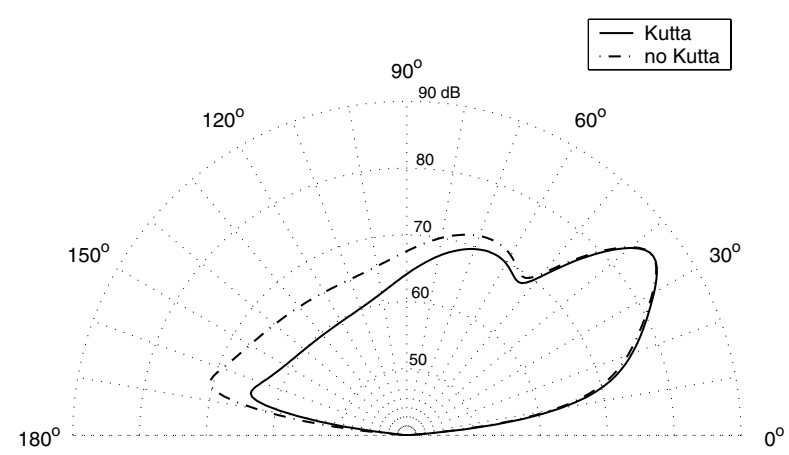

(b) with mean flow

Figure 8. With vs without mean flow for 1st radial mode with $\omega=15$

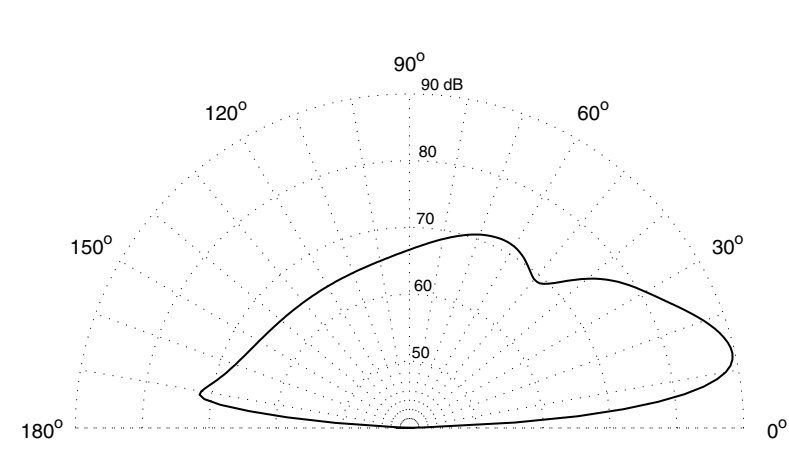

(a) no mean flow

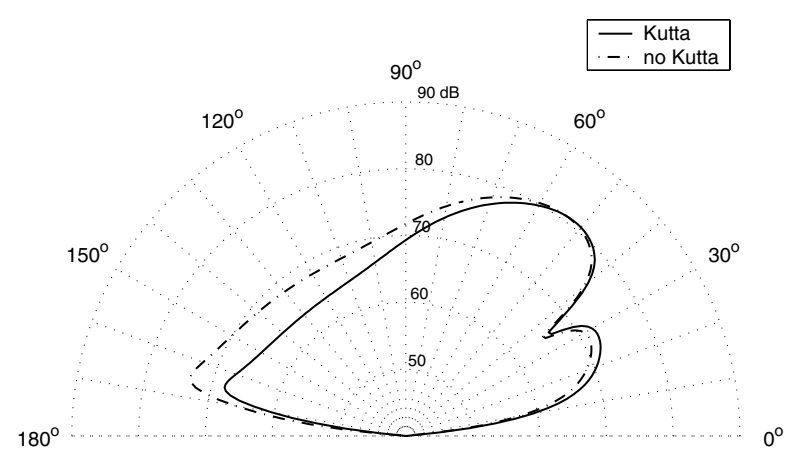

(b) with mean flow

Figure 9. With vs without mean flow for 2 nd radial mode with $\omega=15$

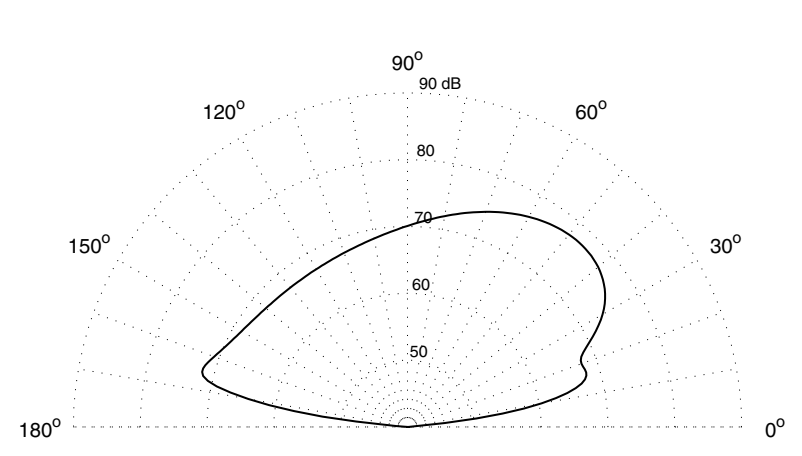

(a) no mean flow

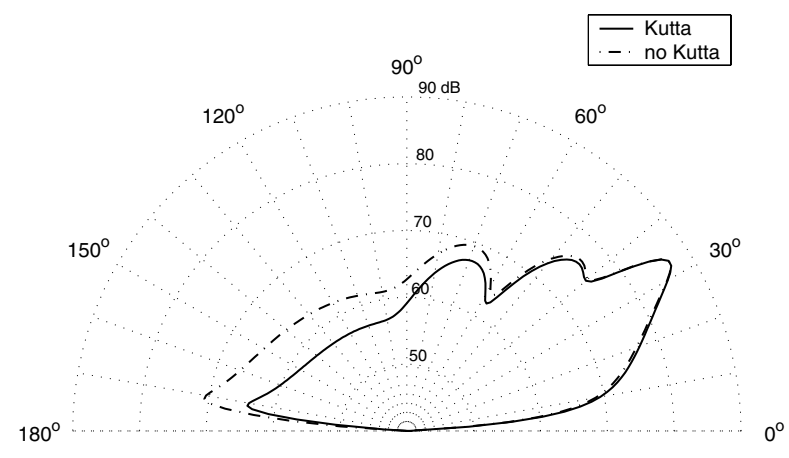

(b) with mean flow

Figure 10. With vs without mean flow for 1st radial mode with $\omega=25$ 


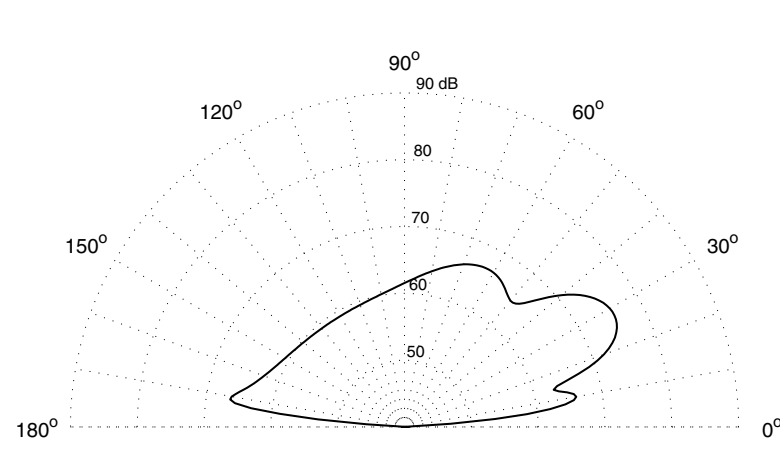

(a) no mean flow

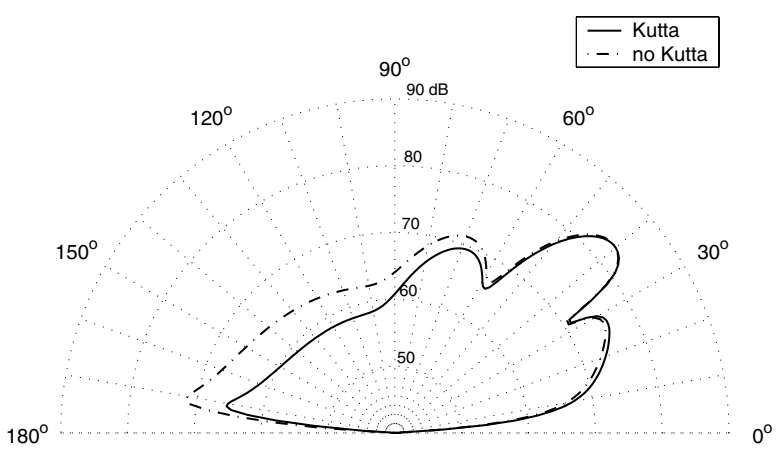

(b) with mean flow

Figure 11. With vs without mean flow for 2 nd radial mode with $\omega=25$

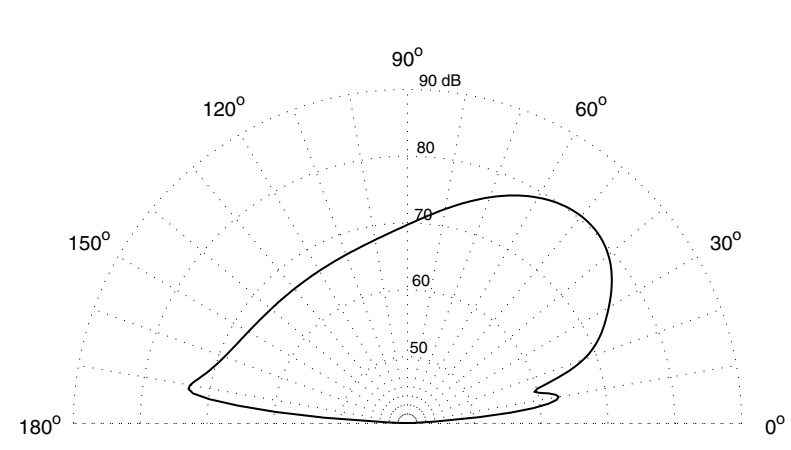

(a) no mean flow

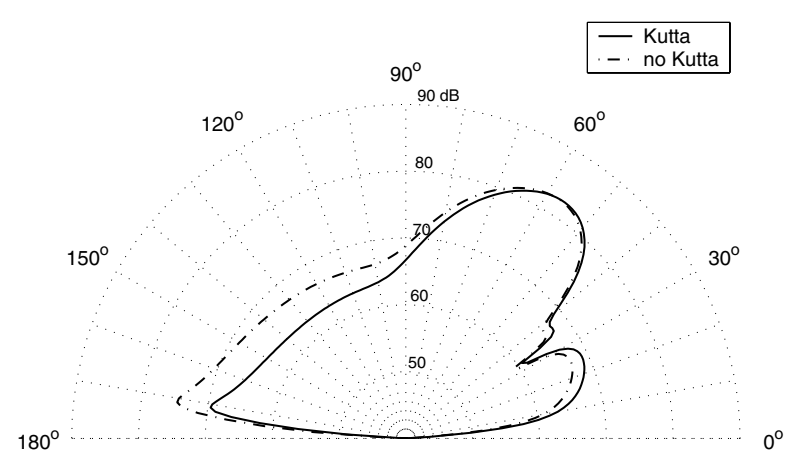

(b) with mean flow

Figure 12. With vs without mean flow for $3 d$ radial mode with $\omega=25$

\section{Near field}

In this section we present the near field of two cases with a lined centerbody, comparing reduced frequencies $\omega=15$ with $\omega=25$. In both cases the first radial mode and full Kutta condition is considered. We see the lobe structure that is also found in the directivities presented above, but in addition we see that for the $\omega=25$-case part of the downstream radiated field remains relatively long captured inside the jet.

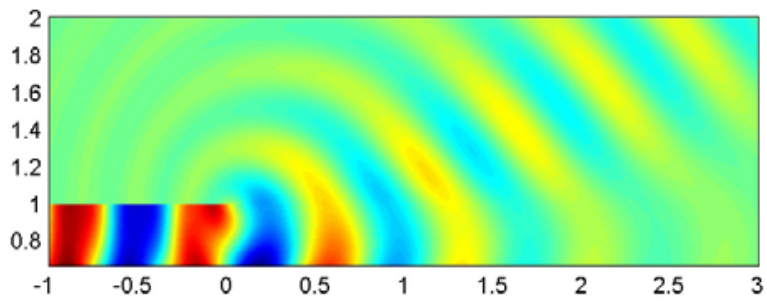

(a) $\omega=15$

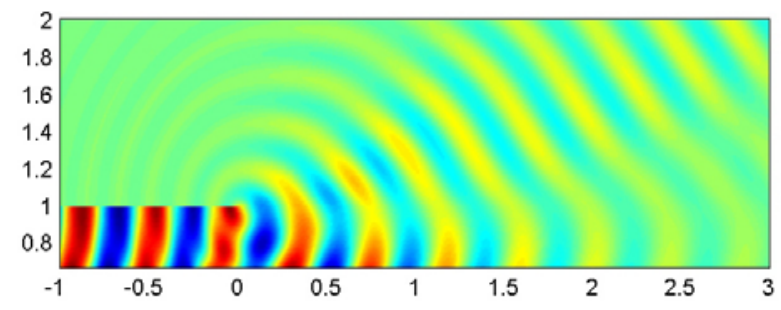

(b) $\omega=25$

Figure 13. Near fields of $\omega=15$ and 25 with full Kutta condition

\section{Lined centerbody vs afterbody}

In this section we compare a fully hard-walled hub with a lined centerbody and a lined afterbody. The configuration is much like before, with mean flow, full Kutta condition and the first radial mode incident. 
We see for the lower frequency $\omega=15$ the highest values for the untreated case ("hard") along most of the angular range, while the fully soft hub ("soft") and the soft afterbody ("hard-soft") yields on average the same attenuation. The lined centerbody is slightly lower in the downstream arc, and the lined afterbody is lower in the upstream arc.

The comparable figure for the higher frequency $\omega=25$ shows a similar behaviour although the apparent interference of scattered modes in crosswise direction leads to a slightly more pronounced lobed structure for the lined afterbody radiation pattern.

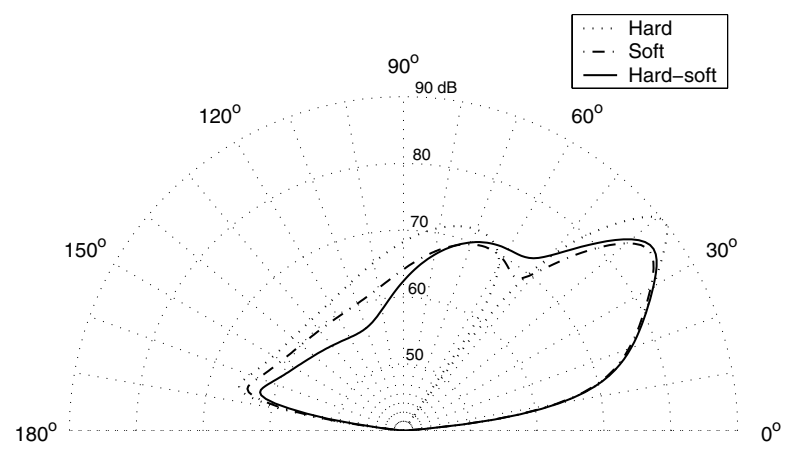

(a) $\omega=15$

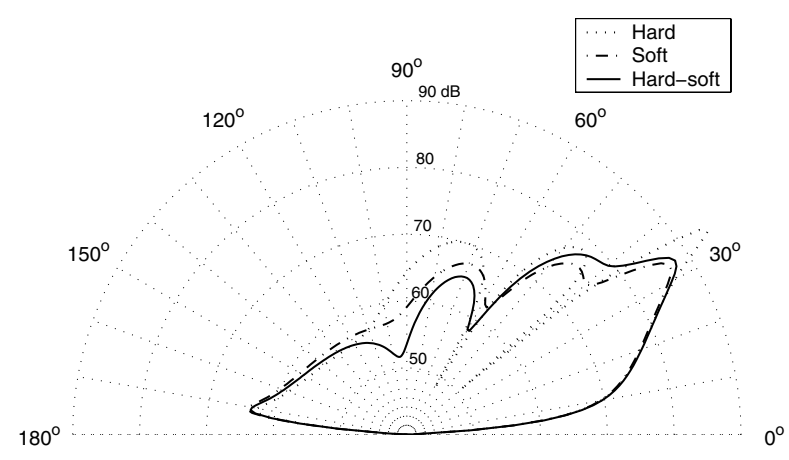

(b) $\omega=25$

Figure 14. Lined centerbody vs afterbody for 1st radial mode with $\omega=15$ and $\omega=25$

\section{Appendix}

Since $\log (K(u)) \sim \pm \log |u|$ for $|u| \rightarrow \infty$ ('+' with mean flow, '-' without), the integral (19b) for the split functions does not converge normally at infinity, and we have to convert it into a Cauchy Principle Value integral [13, p.42]. We take the limit symmetrically around a suitable real number between the branch points of $\lambda_{0}$ and $\lambda_{1}$ that separates the right and left running modes (this is not always possible, in which case the contribution of the missed poles have to be added as residues; see [7,8]). Without going into every detail, we obtain typically a contour as depicted in figure 15. More details can be found in [17]. A very important point that has to be checked is the question whether anywhere along the contour $K(u)$ crosses the branch cut of the logarithm function, which is normally chosen along the negative real axis. In such a case the integrand is not analytic and the split integral is invalid. An example of the behaviour of $K(u)$ is shown in figure 16 .

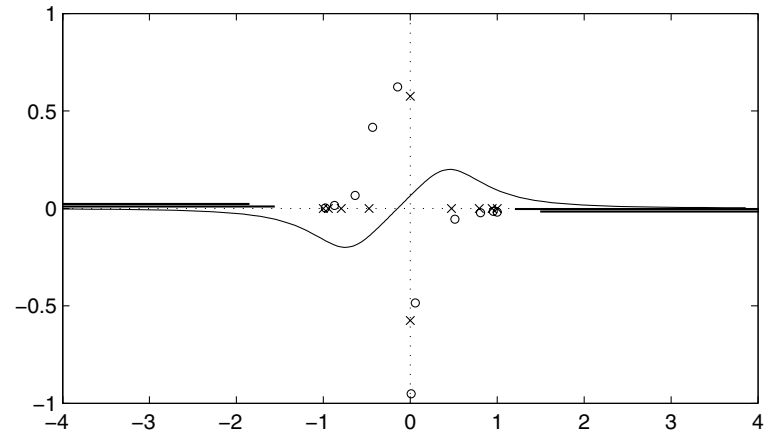

Figure 15. Sketch of deformed integration contour in $u$-plane.

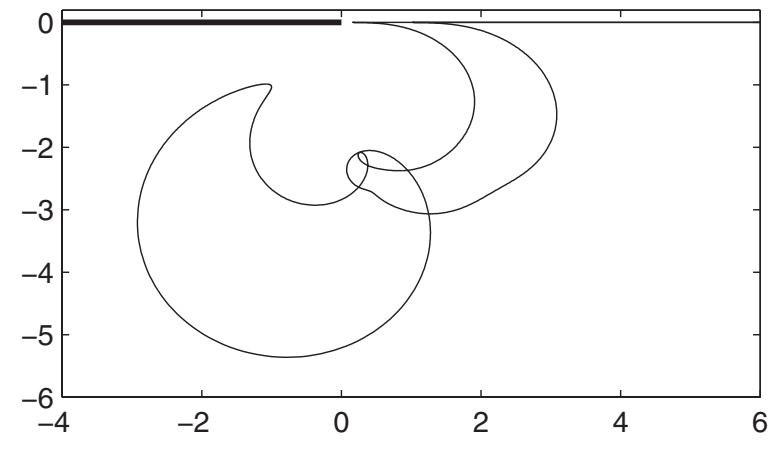

Figure 16. Tracing $K(u)$ in the complex plane when $u$ varies along the contour. Note that it never crosses the negative real axis, and indeed $K(u) \sim|u|$ for large $|u| \cdot M_{j}=0.2, M_{O}=0, h=0, \omega=20$, $m=4, D_{1}=C_{1}=1$. 


\section{Conclusions}

Analytical solutions for the problem of radiation of sound from a duct have been shown to be possible only for relatively idealized configurations, for example the seminal Wiener-Hopf solution by Levine and Schwinger for the semi-infinite circular unflanged duct of infinitely thin hard walls. It was shown by Carrier that this classical problem without mean flow can be extended to include a subsonic mean flow, although in the case of an exhaust flow the issue of the instability of the jet and the Kutta condition at the trailing edge was only resolved by Munt. He showed that application of the Kutta condition, in order to determine the amount of vorticity shed from the trailing edge, goes together with excitation of the Kelvin- Helmholtz instability wave of the jet. This is particularly relevant for numerical solutions in time domain, as these instabilities are practically difficult to avoid.

For many engine-aircraft engineering applications this problem has served as an important first model, useful for understanding the physics and for validating and benchmarking numerical solutions. Exactly for this reason Munt's solution was recently generalized by Gabard e.a. to include a doubly infinite hard-walled centerbody or hub.

In the present work we have extended this work further by considering the effect of lining on the centerbody and on the afterbody. The lining is of impedance type, while the Ingard-Myers boundary condition is taken to include the effect of the mean flow. Any instability of the vortex sheet along the lined wall is ignored, and considered as probably being unphysical. The flow is assumed to be subsonic. Differences in the (otherwise uniform) mean flow velocity, density and temperature are taken into account. A vortex sheet which separates the jet from the outer flow emanates from the edge. Due to this velocity discontinuity of the mean flow the jet is unstable. An analytical solution satisfying the full or partial Kutta condition at the trailing edge is found by means of the Wiener-Hopf technique. The jet instability wave is taken apart from the rest of the solution and examples, both in far field and near field, are given to illustrate the found solutions. The effect of the Kutta condition, the mean flow and the lining is shown graphically. Some effect of the hard-soft transition in the lined afterbody case is given, but conclusions cannot yet be drawn.

The problem of a lined afterbody is of particular interest, because its Wiener-Hopf solution requires so-called "weak factorization". In the present context of radiation from a jet exhaust this is a novel application of this already rather old technique.

\section{Acknowledgements}

This work was carried out under the European collaborative project "TURNEX" under the Sixth Framework Programme (Technical Officer Daniel Chiron, Project Coordinator Brian Tester). The financial support is greatly acknowledged. The authors would like to express their appreciation for the interest and fruitful discussions with Gwenael Gabard (ISVR) and the partners of the project.

\section{References}

${ }^{1}$ H. Levine and J. Schwinger. On the radiation of sound from an unflanged circular pipe. Physical Review, 73:383-406, 1948

${ }^{2}$ G.F. Carrier. Sound transmission from a tube with flow. Quarterly of Applied Mathematics, 13:457-461, 1956.

${ }^{3}$ R.M. Munt. The interaction of sound with a subsonic jet issuing from a semi-infinite cylindrical pipe. Journal of Fluid Mechanics, 83(4):609640, 1977.

${ }^{4}$ X. Zhang, X.X. Chen, C.L. Morfey and B.J. Tester, Computation of Fan Noise Radiation through A Realistic Engine Exhaust Geometry with Flow, AIAA 2003-3267, 9th AIAA/CEAS Aeroacoustics Conference 12-14 May 2003

${ }^{5}$ X. Zhang, X. Chen, C.L. Morfey, P.A. Nelson. Spinning Modal Radiation from an Unflanged Duct . AIAA Paper, $2002-2475$ 2002, 8th AIAA/CEAS Aeroacoustics Conference, Breckenridge.

${ }^{6}$ X. Zhang, X. Chen, C.L. Morfey, P.A. Nelson. Infinite Duct with a Subsonic Jet. AIAA Paper, 2003-08812003, 41st AIAA Aerospace Sciences Meeting, Reno

${ }^{7}$ G. Gabard, R. Astley, M. Ben Tahar Noise Radiation from a Jet Pipe: A Benchmark Problem for Computational Aeroacoustics AIAA-20053064 11th AIAA/CEAS Aeroacoustics Conference, 23-25 May 2005

${ }^{8}$ G. Gabard, R.J. Astley, Theoretical models for sound radiation from annular jet pipes: far- and near-field solutions, Journal of Fluid Mechanics, 549:315-342, 2006

${ }^{9}$ K.U. Ingard, Influence of Fluid Motion Past a Plane Boundary on Sound Reflection, Absorption, and Transmission, Journal of the Acoustical Society of America 31(7), 1035-1036, 1959

${ }^{10}$ M.K. Myers, On the acoustic boundary condition in the presence of flow, Journal of Sound and Vibration, 71 (3), p.429-434, 1980

${ }^{11} \mathrm{M}$. Idemen, A new method to obtain exact solutions of vector Wiener-Hopf equations, ZAMM 59:656-658, 1979

${ }^{12}$ I.D. Abrahams, Scattering of sound by two parallel semi-infinite screens, Wave Motion 9:289-300, 1987

${ }^{13}$ B. Noble, Methods based on the Wiener-Hopf Technique, Pergamon Press, London, 1958.

${ }^{14}$ A. Büyükaksoy, A. Demir, Radiation of sound from a semi-infinite rigid duct inserted axially into a larger infinite tube with wall impedance discontinuity, ZAMM (in press) 
${ }^{15}$ S.W. Rienstra, Impedance Models in Time Domain including the Extended Helmholtz Resonator Model, AIAA 2006-2686 of the 12th AIAA/CEAS Aeroacoustics Conference, Cambridge, MA, 8-10 May 2006

${ }^{16}$ S.W. Rienstra and B.J. Tester, An Analytic Green's Function for a Lined Circular Duct Containing Uniform Mean Flow, AIAA 2005-3020 of the 11th AIAA/CEAS Aeroacoustics Conference, Monterey, CA, 23-25 May 2005

${ }^{17}$ S.W. Rienstra and N. Peake, Modal Scattering at an Impedance Transition in a Lined Flow Duct, AIAA 2005-2852 of the 11th AIAA/CEAS Aeroacoustics Conference, Monterey, CA, 23-25 May 2005

${ }^{18}$ S.W. Rienstra, Acoustic Radiation From A Semi-Infinite Annular Duct In A Uniform Subsonic Mean Flow, Journal of Sound Vibration, 94(2):267-288, 1984 